\title{
A construção do conhecimento na odontologia: a produção científica em debate
}

\author{
Karla Patrícia Cardoso Amorim², Maria do Socorro Costa Feitosa Alves ${ }^{3}$, Raimunda Medeiros Germano ${ }^{4}$
}

Amorim KPC, Alves MSCF, Germano RM. A construção do conhecimento na odontologia: a produção científica em debate. Acta Cir Bras [serial on line] Available from: URL: htt://www.scielo.br/acb.

RESUMO - Objetivo: Analisar, quantitativamente, que revistas odontológicas estão sendo consultadas e lidas por cirurgiõesdentistas, e avaliar os perfis das mais citadas. Métodos: Foram realizadas 370 pesquisas de opinião, durante o XVII Congresso Pernambucano de Odontologia e o IX Congresso de Odontologia do Rio Grande do Norte, realizados, respectivamente, em abril e setembro de 2004. Os participantes eram profissionais voluntários divididos entre a clínica geral (37,84\%) e dez especialidades distintas (62,16\%); onde 77,02\% possuíam até 10 anos de formados. Resultados: Proveniente das pesquisas realizadas, 620 citações foram geradas; nas quais, 32 revistas diferentes, nacionais e internacionais, foram citadas. Analisando os dados coletados, observamos que três revistas totalizaram mais da metade das citações (52,74\%), quais sejam: Revista da Associação Paulista de Cirurgiões-Dentistas - APCD, Revista da Associação Brasileira de Odontologia - ABO nacional e a Revista Brasileira de Odontologia-RBO. Conclusão: Apesar dos participantes da pesquisa serem, na maioria, especialistas, observamos uma tendência de maiores consultas às revistas de caráter geral. Essas revistas abordam predominantemente assuntos clínicos e técnicos e apresentam tiragem expressiva, grande penetração no meio odontológico, fácil acessibilidade e muito tempo de mercado (duas delas com mais de 50 anos). Pela avaliação da CAPES - QUALIS, todas possuem conceito B nacional. Dessa forma, faz-se necessário um aprofundamento desse estudo, a fim de analisarmos qualitativamente que tipo de assuntos e temáticas essas revistas abordam, uma vez que influenciam na formação e prática odontológica.

DESCRITORES: Revistas científicas. Revistas odontológicas. Avaliação das revistas odontológicas.

\section{Introdução}

A forma como o saber é construído e divulgado é de suma importância, uma vez que irá influenciar e nortear os pensamento, reflexões e as atitudes, moldando os fazeres em todos os campos do conhecimento.

As revistas são formas dinâmicas de divulgar o conhecimento. Refletindo, criticamente, observamos que as revistas científicas na área da odontologia, bem como nas demais área do saber, não têm apenas o propósito de estabelecer um elo de comunicação entre pesquisadores, profissionais e estudantes de odontologia. Elas servem de depositárias das concepções que vão plasmando, moldando e dando existência à odontologia nacional e à ciência.

Atualmente, são publicados mais de 600 mil revistas científicas em todo o mundo, estimando-se que sejam escritos, diariamente, entre seis e sete mil artigos científicos para alimentá-las ${ }^{2}$.

A importância de se pesquisar sobre as revistas científicas é fortalecida pelo pensamento de Dionne e Laville, quando afirmam que são nelas que se vê melhor e mais rapidamente a ciência que se faz, e a comunidade pode avaliar a justa medida da pesquisa, pois o pesquisador precisa dizer o essencial, e com concisão, pois as páginas são limitadas ${ }^{3}$.Uma pesquisa, sobre o tempo de vida das revistas nacionais na área de odontologia, aponta que são poucas revistas que podem ser consideradas sólidas e tradicionais ${ }^{4}$. Devido a esses fatores, o presente estudo tem como objetivo analisar, quantita- tivamente, que revistas odontológicas estão sendo lidas e/ ou consultadas por cirurgiões-dentistas, e avaliar os perfis das mais apontadas.

Pesquisas a respeito desse assunto e temática são escassas na área odontológica; assim sendo, o presente estudo irá contribuir para que se repense sobre a importância que os periódicos desempenha no contexto atual da ciência e da vida.

\section{Métodos}

A presente pesquisa tem caráter exploratório descritivo, dentro de uma abordagem quantitativa.

Foram realizadas 370 pesquisas de opinião, com o intuito de apreender que revistas os cirurgiões-dentistas estão utilizando como fonte de conhecimento, a fim de avaliar os perfis das mais apontadas. Esses dados foram coletados durante dois eventos realizados pela Associação Brasileira de Odontologia - ABO, no ano de 2004: o XVII Congresso Pernambucano de Odontologia, realizado em Recife no mês de abril e o IX Congresso de Odontologia do Rio Grande do Norte, realizado em Natal no mês de setembro. Essa coleta se deu em locais de grande circulação nos referidos eventos.

Os participantes, respondentes da pesquisa, foram escolhidos de maneira aleatória e voluntária, após explicação dos objetivos do estudo. Ao final, totalizaram uma amostra de 370

1. Pesquisa realizada no Programa de Pós-Graduação em Ciências da Saúde da Universidade Federal do Rio Grande do Norte.

2. Doutoranda do Programa de Pós-Graduação em Ciências da Saúde - UFRN e Mestre em Odontologia Social - UFRN.

3. Doutora em Odontologia Preventiva e Social - FOP-UPE e Professora Adjunto do Programa de Pós-Graduação em Ciências da Saúde da UFRN.

4. Doutora em Educação pela UNICAMP e Professora Adjunto e Vice-coordenadora do Programa de Pós-Graduação em Enfermagem - UFRN. 
profissionais. A única condição exigida, para serem incluídos na pesquisa, era que fossem cirurgiões-dentistas formados. A esses foi feita a seguinte pergunta por escrito: Qual(is) a(s) revista(s) na área odontológica que você mais lê e/ou consulta? Os respondestes podiam citar quantas revistas quisessem.

$\mathrm{Na}$ ficha de resposta também incluímos a especialidade e tempo de formado, a fim de fazermos uma breve caracterização dos respondentes.

\section{Resultados}

Foram obtidas 620 citações, a partir das pesquisas realizadas com os 370 cirurgiões-dentista. Desses, 140 eram clínicos gerais $(37,84 \%)$, e 230 especialistas $(62,16 \%)$ de dez especia- lidades distintas, quais sejam: endodontistas (44), ortodontista (38), protesistas (40), odontopediatras (30), especialistas em dentística (29), cirurgiões buço-maxilofaciais (25), estomatologistas (9), implantodontistas (5), periodontistas (8), radiologistas (2). Vale ressaltar que 77,02 desses cirurgiões-dentistas possuíam até 10 anos de formados; no entanto, também participaram profissionais com até 35 anos de profissão.

Proveniente das 620 citações, foram evocados os nomes de 32 revistas diferentes, tanto nacionais como internacionais, estas em proporção menor, conforme tabela abaixo, por ordem decrescente de vezes citada:

TABELA 1 - Resultados da pesquisa de opinião sobre as revistas odontológicas mais lidas

\begin{tabular}{|c|c|c|}
\hline REVISTA CITADAS & $\mathrm{N}^{\circ} \mathrm{X}$ & $\%$ \\
\hline Rev da Assoc Paulista de Cirurgiões-dentistas - APCD & 182 & 29,35 \\
\hline Rev da ABO nacional & 97 & 15,64 \\
\hline Rev Brasileira de Odontologia - RBO & 48 & 7,74 \\
\hline Jornal Brasileiro de Endodontia - JBE & 35 & 5,64 \\
\hline The Journal of the American Dental Association - JADA & 21 & 3,40 \\
\hline Jornal Brasileiro de Ortodontia e Ortopedia Facial & 21 & 3,40 \\
\hline Jornal Brasileiro de Clínica Integrada - JBC & 20 & 3,22 \\
\hline Rev Ibero-Americana de Estética e Dentística - JBD & 18 & 2,90 \\
\hline Rev Prótese Clínica e Laboratorial - PCL & 18 & 2,90 \\
\hline Dental Press Ortodontia & 18 & 2,90 \\
\hline Pesquisa Odontológica Brasileira - POB & 17 & 2,74 \\
\hline Rev da Pós-graduação da FOUSP - RPG & 17 & 2,74 \\
\hline Rev Internacional de Periodontia Clínica-RPE & 13 & 2,10 \\
\hline Rev Ibero-Americana de Odontopediatria e Odontologia do Bebê - JBP & 13 & 2,10 \\
\hline Revista Gaúcha de Odontologia - RGO & 12 & 1,93 \\
\hline Revista de odontologia da UNESP & 09 & 1,45 \\
\hline Brazilian Endodntic Journal & 08 & 1,30 \\
\hline Rev Brasileira de Cirurgia e Implantodontia - BCI & 07 & 1,12 \\
\hline Brazilian Dental Journal & 07 & 1,12 \\
\hline Brazilian Oral Research & 06 & 0,97 \\
\hline Rev Internacional de Cirurgia e Traumatologia Bucomaxilofacial - RBC & 05 & 0,80 \\
\hline Quitessence Internacional & 04 & 0,64 \\
\hline Oral Surgery, Oral Medicine, Oral Pathology & 04 & 0,64 \\
\hline Revista Paulista de Ortodontia & 03 & 0,50 \\
\hline Oral Oncology & 03 & 0,50 \\
\hline Rev Brasileira de Implantodontia e Prótese sobre Implante - RBP & 03 & 0,50 \\
\hline Oral Diseases & 02 & 0,32 \\
\hline Journal of Orofacial Pain & 02 & 0,32 \\
\hline Odonto Ciência & 02 & 0,32 \\
\hline Journal of Prothetic Dentistry & 02 & 0,32 \\
\hline Rev da Associação Brasileira de Radiologia Odontológica - ABRO & 02 & 0,32 \\
\hline Journal of Orthodontics & 01 & 0,16 \\
\hline TOTAL & 620 & 100 \\
\hline
\end{tabular}




\section{Discussão}

Diante dos resultados, observamos que as três revistas mais citadas representam mais da metade das respostas; ou seja, menos de $10 \%$ das revistas evocadas contabilizam 52,74\% das citações. Tais resultados, de uma certa forma, estão de acordo com os descritos por King e Tenopir, quando afirmam que poucas revistas são consultadas com grande freqüência, e muitas revistas sã lidas poucas vezes ${ }^{5}$.

A questão da acessibilidade aos periódicos é um fator de peso. A Revista APCD e a Revista ABO nacional, as duas mais citadas, trabalham esse fator de uma forma positiva. A primeira é remetida gratuitamente aos associados da Associação Paulista de Cirurgiões-Dentistas, que totalizam mais de 36.000; a segunda, é disponibilizada gratuitamente aos associados da Associação Brasileira de Odontologia Nacional, suas 27 Seções estaduais e 288 Regionais.

Esse aspecto também é abordado por King e Tenopir, quando ressaltam que os cientistas fazem as suas escolha em relação à assinatura e compra de periódicos segundo a lógica de mercado. Assinam revistas de preços baixos que lêem com frequiência, e consultam bibliotecas para ter acesso a revistas caras que lêem ocasionalmente ${ }^{5}$.

As três revistas mais citadas possuem caráter geral; ou seja, atingirem do clínico geral ao especialista. É importante frisar, que essas abordam, predominantemente, assuntos e temáticas de âmbito clínico e técnico; e como bem sabemos o perfil da odontologia brasileira ainda é marcado pela ênfase ao biológico e técnico-restaurador. Perfil este, que de uma certa forma, se comprova pelas respostas obtidas; uma vez que, os participantes não citaram nenhuma revista na área da saúde coletiva e áreas afins; ou seja, produza conhecimento que alimentem o debate sobre grandes questões conceituais e teóricas fundamentais, fotalecendo abordagens que compreendam a saúde como componente relevante da vida e da organização da sociedade ${ }^{6}$.

Analisando o tempo de existência das três primeiras revistas, desde a primeira publicação, observamos que se tratam de periódicos com um certo tempo de circulação: revista APCD - 58 anos, revista da ABO nacional - 12 anos e a RBO 62 anos. Dentro do contexto odontológico, esse fator tem grade importância; visto que existe uma tendência das revistas odontológicas não durarem muito, como bem ressalta Madeira e Carvalho. Esses autores relatam um percentual de interrupção de 73,25 dos títulos já editados na área de odontologia até o ano de 1988. Essa questão interfere na credibilidade da revis$\mathrm{ta}^{4}$.

A questão da credibilidade é sentida, ao analisarmos pesquisas a respeito de revistas odontológicas. São poucas as pesquisas da área que versam sobre tal assunto; no entanto, das que abordam, grande parte incluem a Revista APCD e a RBO. Louro Filho (1982) utilizou, no trabalho Avaliação e Perpectivas 1982 - Odontologia, realizado a pedido do Conselho Nacional de Desenvolvimento Científico e Tecnológico (CNPq), uma amostra contendo nove periódico odontológicos brasileiros, dentre os quais faziam parte a APCD e a RBO .

10 - Acta Cirúrgica Brasileira - Vol 20 - Supl no 12005
Narvai, em sua tese de doutorado, sobre a produção científica na área da odontologia preventiva e social, utilizou 19 revista, onde novamente fizeram parte as duas revistas citadas. ${ }^{8}$ O trabalho sobre a vida das revistas odontológicas, referido anteriormente, também as citam ${ }^{4}$.

Com relação à tiragem, vemos que tanto a revista $\mathrm{APCD}$, como a $\mathrm{ABO}$ nacional têm uma grande tiragem; correspondendo, respectivamente, a 43.000 e 30.000 exemplares. Já, a RBO possui um número menor, 7.000 exemplares.

Fazendo uma relação da tiragem versus periodicidade das revistas em questão, vemos que as três tratam-se de publicações bimestrais, com periodicidade regular. Dessa forma, concluímos que possuem um grande poder de penetração, principalmente as duas primeiras; visto que, em média circulam, anualmente, 258.000 exemplares da APCD e 180.000 exemplares da $\mathrm{ABO}$ nacional.

$\mathrm{O}$ fato das revistas APCD, ABO nacional e RBO, estarem classificadas com conceito B nacional, pela avaliação da fundação CAPES (Coordenação de Aperfeiçoamento de Pessoal de nível Superior) - QUALIS, não impediu de serem as mais citadas; ou seja, as mais lidas e/ou consultadas pelos dentistas. Grande parte das revistas odontológicas não preenchem os critérios de impacto, da competitividade e da internacionalidade, tão difundidos atualmente no mundo científico . Esses aspectos, colocam algumas questões de difícil equacionamento, tais como os critérios utilizados pelas bases de dados, a língua na qual os trabalhos são produzidos, a concentração de conhecimentos em nações economicamente mais desenvolvidas. Tais questionamentos se potencializam, se considerarmos o caso de nações periféricas como é o caso do Brasil ${ }^{10}$.

No entanto, sabemos que critérios de avaliação têm que existir e são de extrema importância, porém como ressalta Amorim, o assunto é complexo, e os critérios muito vagos ${ }^{11}$.

A questão levantada com os resultados da presente pesquisa é que tais critérios de avaliação dos periódicos, parece influenciar mais diretamente os pesquisadores que os leitores; revelando, dessa forma, que outros fatores influenciam na escolha das leituras realizadas pelos cirurgiões-dentistas.

\section{Conclusão}

Apesar dos participantes da pesquisa serem, na maioria, especialistas, observamos uma tendência de maiores consultas e leituras às revistas de caráter geral. Essas revistas abordam predominantemente assuntos clínicos e técnicos.

As revistas mais reportadas apresentam tiragem expressiva, grande penetração no meio odontológico, fácil acessibilidade e muito tempo de mercado (duas delas com mais de 50 anos). Pelo programa de avaliação da CAPES (QUALIS), todas possuem conceito $\mathrm{B}$ nacional.

Dessa forma, faz-se necessário um aprofundamento desse estudo, a fim de analisarmos qualitativamente que tipo de 
assuntos e temáticas essas revistas abordam; uma vez que, influenciam na formação e prática odontológica.

\section{Referências}

1. Biojone M R. Forma e função dos periódicos científicos na comunicação da ciência [Dissertação - Mestrado]. Universidade de São Paulo - Escola de Comunicação e Artes; 2001.

2. Trzesniak P A. Concepção e a construção da revista científica. In: Curso de Editoração Científica. Petrópolis: ABEC; 2001. p.17-23

3. Laville C, Dionne J. A construção do Saber - Manual de metodologia da pesquisa em ciências humanas. Porto Alegre: Editora UFMG; 1999.

4. Madeira M C, Carvalho M A. Tempo de vida das revistas nacionais de odontologia. RGO 1988;4:301-6.

5. King D W, Tenopir C. A publicação de revistas eletrônicas: economia da produção, distribuição e uso. Ciência Inform 1998;2:176-82.
6. Barreto M L. O conhecimento científico e tecnológico como evidência para políticas e atividades regulatórias em saúde. Ciên $\mathrm{S}$ Col 2004;2:329-38.

7. Louro Filho P P..Odontologia. In: SEPLAN. CNPq.. Avaliação e perspectivas. Ciências da Saúde 1982;6:437-93.

8. Narvai P C. Produção científica na área de odontologia preventiva social. BRASIL, 1986-1993 [Tese - Doutorado]. Universidade de São Paulo - Faculdade de Saúde Pública; 1997.

9. Foratini O P. A internacionalidade da ciência. Rev Saúde Publica. 1997;2:115.

10. Yamamoto O H, Souza C C, Yamamoto M E. A produção científica na psicologia: uma análise dos periódicos brasileiros no período de 1990-1997. Psicol Reflex Crit. 1999;2:13-5.

11. Amorim R F B. Avaliação dos periódicos científicos: uma necessidade da pesquisa científica brasileira. Rev Bras Patol Oral 2003;4:23-5.

Amorim KPC, Alves MSCF, Germano RM. Construction of knowledge in odontology: the scientific production in debate. Acta Cir Bras [serial on line] Available from: URL: htt://www.scielo.br/acb.

ABSTRACT - Purpose: Analyze, quantitatively, which odontology magazines are being consulted and read by surgeon-dentists, and evaluate the profiles of the mostly cited. Methods: 370 polls were made, during the XVII Congresso Pernambucano de Odontologia (Pernambucan Congress of Odontology) and the IX Congresso de Odontologia do Rio Grande do Norte (Congress of Odontology of Rio Grande do Norte), which took place, respectively, in April and September 2004. The participants were volunteering professionals divided among General Practice (37.84\%) and ten differing specialties (62.16\%); of which 77,02\% had up to ten years of graduation. Results: Resulting the researches made, 620 quotes were generated, in which, 35 different magazines, national and international, were quoted. By analyzing the data collected, we observe that three magazines summed more than half of the quota (52.74\%), which are: Revista da Associação Paulista de Cirurgiões Dentistas - APCP (Magazine of the PaulistaAssociation of Surgeon-Dentists), Revista da Associação Brasileira de Odontologia-ABO national (Magazine of the Brazilian Association of Odontology) and the Revista Brasileira de Odontologia - RBO (Brazilian Magazine of Odontology). Conclusion: Despite the participants of the research being, in their majority, specialists, we observe a tendency of a greater consultation of the magazines of general characteristic. These magazines predominantly tackle clinical and technical subjects and present an expressive circulation, great penetration in the odontologic world, easy accessibility and a long time in the market (two of them are over 50 years-old). According to CAPES - QUALIS, all possess a B national concept. Therefore, it is necessary a deepening of this study, so as to analyze qualitatively what kind of subjects and themes these magazines touch, since they influence the odontologic formation and practice.

KEYWORDS: Scientific magazines. Odontology magazines. Evaluation of the odontology magazines. 